\title{
All-optical on-chip sensor for high refractive index sensing
}

Yazhao Liu and H. W. M. Salemink'

Citation: Appl. Phys. Lett. 106, 031116 (2015); doi: 10.1063/1.4906576

View online: http://dx.doi.org/10.1063/1.4906576

View Table of Contents: http://aip.scitation.org/toc/apl/106/3

Published by the American Institute of Physics

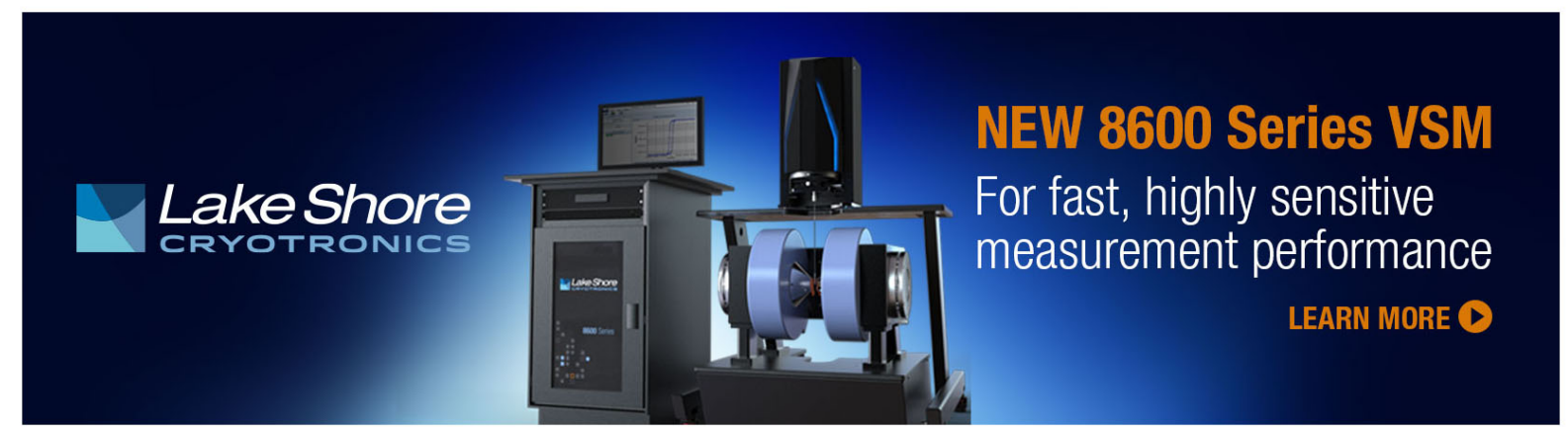




\title{
All-optical on-chip sensor for high refractive index sensing
}

\author{
Yazhao Liu ${ }^{1,2}$ and H. W. M. Salemink ${ }^{3, a)}$ \\ ${ }^{1}$ Foundation for Fundamental Research on Matter, Van Vollenhovenlaan 659, 3527 JP, Utrecht, \\ The Netherlands \\ ${ }^{2}$ Kavli Institute of Nanoscience, Delft University of Technology, Lorentzweg 1, 2628 CJ, Delft, \\ The Netherlands \\ ${ }^{3}$ Institute for Molecules and Materials, Radboud University Nijmegen, Heijendaalseweg 135, \\ 6525 AJ Nijmegen, The Netherlands
}

(Received 19 May 2014; accepted 12 January 2015; published online 22 January 2015)

\begin{abstract}
A highly sensitive sensor design based on two-dimensional photonic crystal cavity is demonstrated. The geometric structure of the cavity is modified to gain a high quality factor, which enables a sensitive refractive index sensing. A group of slots with optimized parameters is created in the cavity. The existence of the slots enhances the light-matter interactions between confined photons and analytes. The interactions result in large wavelength shifts in the transmission spectra and are denoted by high sensitivities. Experiments show that a change in refractive index of $\Delta n \sim 0.12$ between water and oil sample 1 causes a spectral shift of $23.5 \mathrm{~nm}$, and the spectral shift between two oil samples is $5.1 \mathrm{~nm}$ for $\Delta n \sim 0.039$. These results are in good agreement with simulations, which are 21.3 and $7.39 \mathrm{~nm}$ for the same index changes. (C) 2015 Author(s). All article content, except where otherwise noted, is licensed under a Creative Commons Attribution 3.0 Unported License.
\end{abstract}

[http://dx.doi.org/10.1063/1.4906576]

All-optical sensors have attracted much attention in biological/medical research, chemical safety, and environmental monitoring. Unlike electrically interacted sensors, all-optical sensors are free from influences of external electric fields. The principle of operating all-optical sensors relies on variations of optical characters when properties of the surrounding media change. ${ }^{1,2}$ Among currently hot research topics in this field, photonic crystal sensors feature an especially small footprint and high quality factor/volume ${ }^{3,4}$ in integrated labon-chip systems. ${ }^{5-10}$ In many previous works, highly confined photons were obtained in photonic crystal cavities under low refractive index embedding (silicon vs. air), ${ }^{1-14}$ whereas optical sensors are expected to work in a liquid environment in most practical cases. High refractive indices of liquids will unavoidably cause problems of photon leakage. Moreover, considerable spatial overlap between confined photons and analytes is needed to induce clear changes in optical properties. It is a challenge to increase the interacting area without impairing the ability to trap photons. ${ }^{15,16}$

Here, we present our investigation on a modified photonic crystal cavity that allows us to overcome the above difficulties. As will be demonstrated, our sensor is designed to work under high refractive index material infilling of $n=1.5$, whose index is much higher than that of many typical liquids. More important, we enlarge the spatial overlap between the trapped photons and analytes by introducing a group of slots inside the cavity with dimensional and locational optimizations. The maximum quality factor obtained in experiment is greater than 8000, and a wavelength shift of $5.1 \mathrm{~nm}$ under a small refractive index modification of $\sim 0.039$ is observed.

The structure of the photonic crystal cavity is illustrated in Figure 1, consisting of holes being etched through a

a)H.Salemink@science.ru.nl silicon slab. A 2- $\mu$ m-thick silicon dioxide layer supports the silicon slab from underneath. The cavity is surrounded by ten rows of holes in the $\Gamma-K$ direction, and 12 rows of holes in the $\Gamma-M$ direction. The cavity is created by removing three holes in the $\Gamma-K$ direction. As a two-dimensional photonic crystal, the slab confines light by total internal reflection. Analytes cover the top of the photonic crystal and fill in the holes. In order to understand the characteristics of highrefractive-index embedding, we deliberately choose the filling material's refractive index as high as $n=1.5$, and calculate the band structure of such a photonic crystal using the three-dimensional finite different time domain (FDTD)

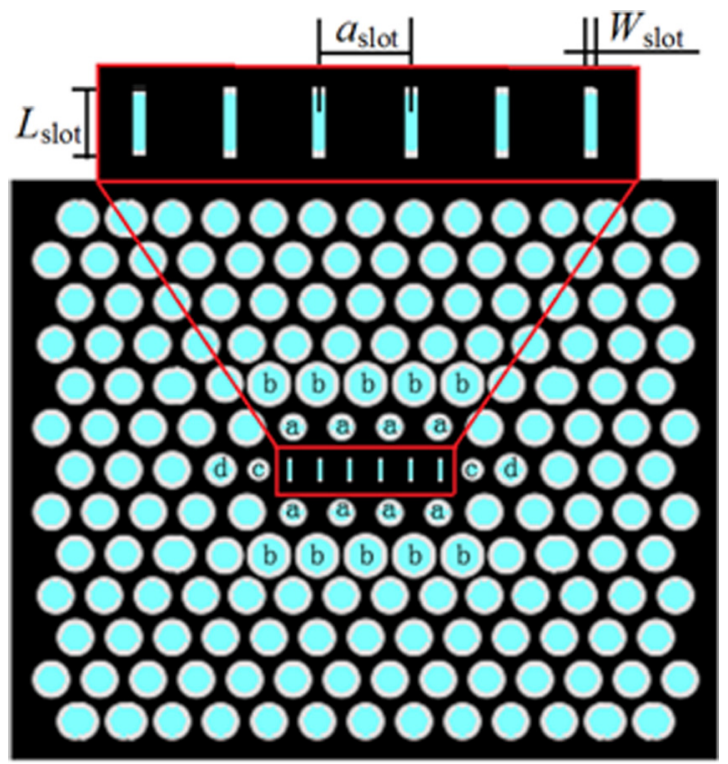

FIG. 1. Photonic crystal cavity with modifications overlaid with a zoomed image of the inside slots. Lattice constant $a=500 \mathrm{~nm}$, radii $r=195 \mathrm{~nm}$; $r_{a}=140 \mathrm{~nm}$ with $\Delta y_{a}=10 \mathrm{~nm}, r_{b}=235 \mathrm{~nm}$ with $\Delta y_{b}=20 \mathrm{~nm}, r_{c}=110 \mathrm{~nm}$ with $\Delta x_{c}=100 \mathrm{~nm}$, and $r_{d}=170 \mathrm{~nm}$ with $\Delta x_{d}=0$. 
simulation program of MEEP. ${ }^{17}$ Unlike typical low-refractive-index embedding, the width of the band gap for highindex embedding becomes much narrower due to a low-refractive-index contrast. Therefore, parameters are set as follows to make the resonant wavelength of the cavity work for near infrared and distant from the band edges. The structure shown in this work has a lattice constant of $a=500 \mathrm{~nm}$. The radii of the holes are set at $r=195 \mathrm{~nm}$. The thickness of the silicon slab is $h=260 \mathrm{~nm}$.

According to previous research on high-Q cavity design, light confinement can be optimized by varying the geometric parameters of the holes surrounding the cavity. ${ }^{12,14}$ We do so by computing resonant frequencies and quality factors of the cavity using the software of harmonic inversion (the built-in "harminv" in MEEP), ${ }^{18}$ which decouple the cavity fields into individual sinusoids and calculate their decay rates. We select the resonant wavelengths with the highest $\mathrm{Q}$ factor after 400 time periods running after an excitation in our simulations. Higher quality factors can be achieved with longer calculation times, but we believe 400 time periods are sufficient.
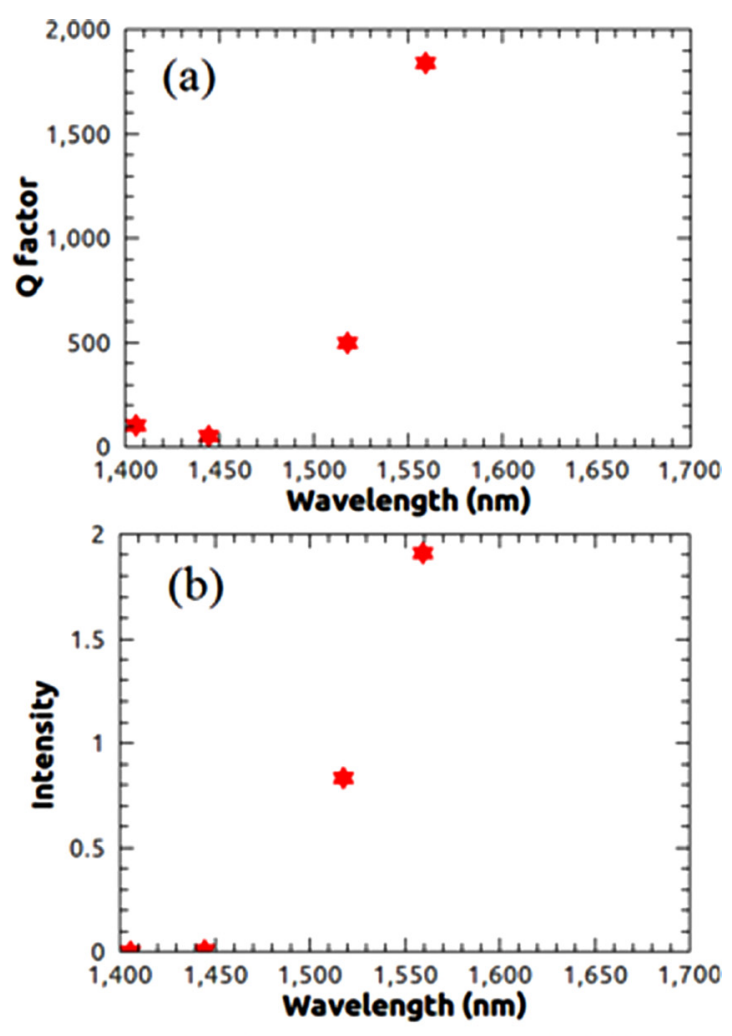

(c)

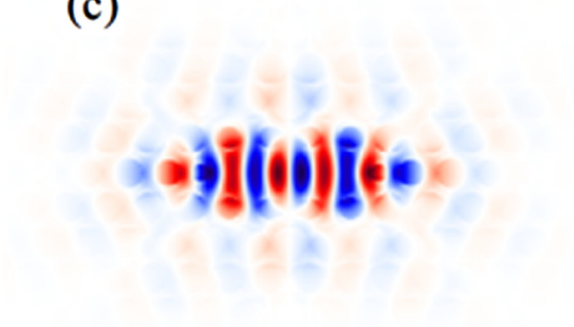

FIG. 2. Quality factors (a), intensities (b), and light distribution (c) of the cavity without inside slots.
The width of the cavity is modified by locally shifting two neighboring rows of holes slightly away from the center in the $\Gamma-M$ direction, with shifting distances of 10 and $20 \mathrm{~nm}$. The first neighboring row has radii of $r_{a}=140 \mathrm{~nm}$ and the second one has enlarged radii of $r_{b}=235 \mathrm{~nm}$. Modifications are also made on the holes in the $\Gamma-K$ direction. The nearest pair of holes is shifted $100 \mathrm{~nm}$ outwardly and reduced to $r_{c}=110 \mathrm{~nm}$. The next pair also has reduced radii of $r_{d}=170 \mathrm{~nm}$.

Only one resonant frequency of $1560 \mathrm{~nm}$ was found with a quality factor greater than 500 . Its Q factor is 1840 with an enhanced intensity of 1.91 . The profile of the electromagnetic-field distribution of the resonant optical mode is obtained by placing a narrow band pulse centered at $1560 \mathrm{~nm}$ in the cavity as a TE-polarized dipole source with Gaussian frequency distribution. As shown in Fig. 2(c), photons are accumulated inside the cavity and symmetrically extended to the nearest-neighboring areas. The in-plane resonant mode has a profile with eight main lobes in the $\Gamma-K$ direction but none in the $\Gamma-M$ direction. In order to enhance the light-matter interaction, we create larger overlap areas for photons and analytes. Unlike our previous work of adding circular holes, ${ }^{19}$ we introduce a group of slots into the cavity. These slots have geometric shapes matching the light profile. Analytes fill in these slots and affect the performance of resonant modes. We use three parameters to adjust the dimensions of the slots, as illustrated in Fig. 1, the periodicity $a_{\text {slot }}$, length $L_{\text {slot }}$, and width $W_{\text {slot }}$. Three-dimensional simulations of quality factors and intensities are performed to examine the effect of introducing slots. All dimensions are normalized to the lattice constant $a$.

Starting with the periodicity, we varied the periodicity $a_{\text {slot }}$ from $0.48 a$ to $0.58 a$ with fixed $L_{\text {slot }}$ and $W_{\text {slot }}$ values of $0.12 a$ and $0.4 a$. We chose the range of variation deliberately by ensuring that $a_{\text {slot }}$ is approximate to the periodicity of the main lobes in Fig. 2(c). As seen in Fig. 3, the quality factor increases from $0.48 a$ and reaches its maxima at $0.53 a$, followed by a sharp decrease. It is easy to understand that confined resonant light forms a stationary-like wave inside the cavity. To keep quality factors high, the slots must have an optimized location with minimal disturbance to the stationary wave.

These optimizations were also applied to the other two parameters $L_{\text {slot }}$ and $W_{\text {slot }}$. We kept $W_{\text {slot }}$ at the same value of $0.4 a$ and varied $L_{\text {slot }}$ from $0.06 a$ to $0.16 a$. The quality factor also decreases sharply as the width increases. It is also easy to see that light confinement inside the cavity depends strongly on the overlap between the light and the slots. Although having the same periodicity, wider slots have larger dimensions than narrower slots, which unavoidably impedes photon confinement and accumulation. Based on similar principles, the $\mathrm{Q}$ factor decreases with increasing $W_{\text {slot }}$ values.

It should be noted that frequencies of resonant modes have slight shifts as the three parameters are adjusted. This clearly indicates that variations of the effective refractive index are caused by different spatial modal distributions. In addition, the quality factors as well as the intensity are lower than the original cavity with no slots, due to the lowered refractive index contrast between the cavity and its neighbors. 

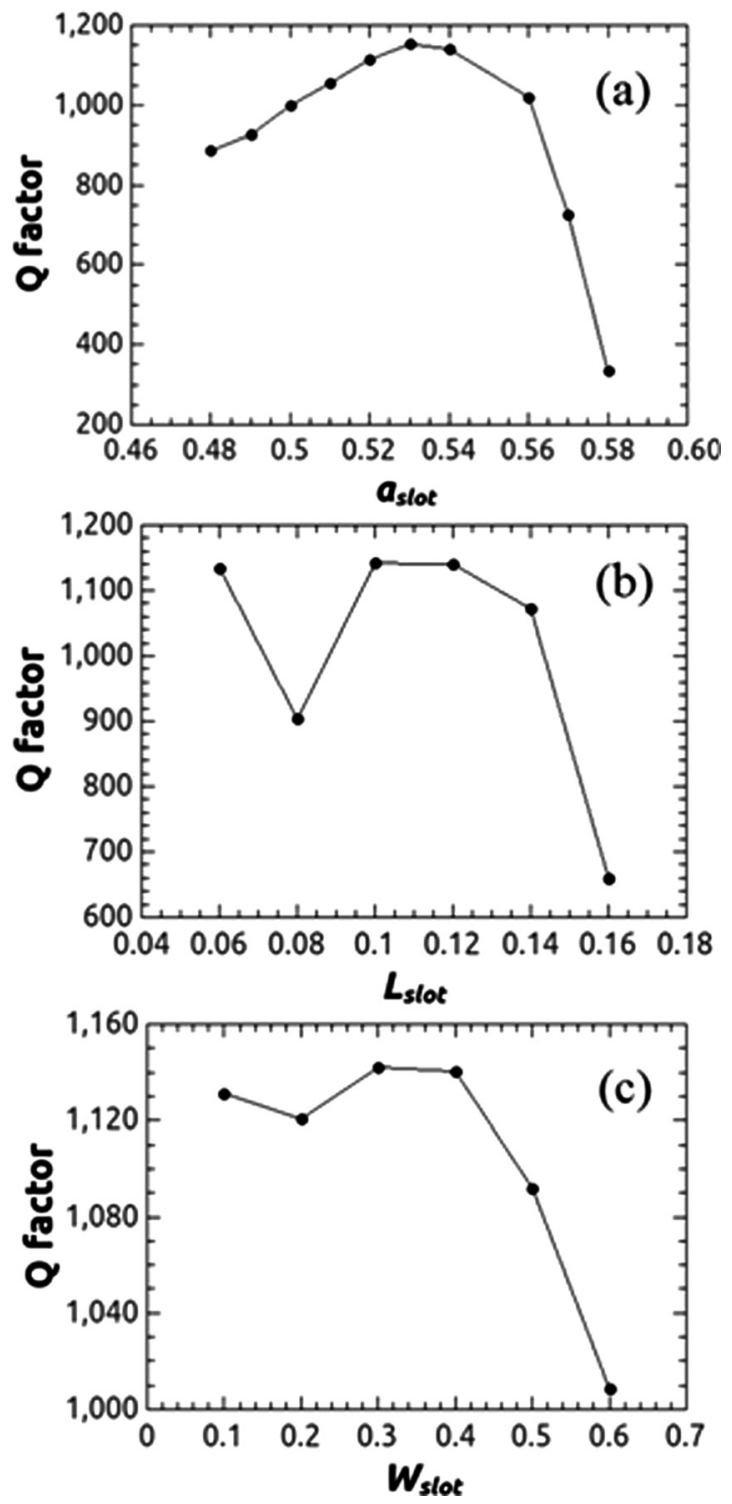

FIG. 3. Relationships between the quality factor and the slot parameters $a_{\text {slot }}$ (a), $L_{\text {slot }}$ (b), and $W_{\text {slot }}(\mathrm{c})$.

With these modifications, we have optimized parameters of the cavity and the best parameters of the inside slots. We understand that a higher $\mathrm{Q}$ factor can be achieved by further modifying the geometry. However, in this work, we have chosen our sensor design as a compromise between high $\mathrm{Q}$ factors and strong light-matter interaction.

To illustrate the operating principle of the sensor and to estimate its sensitivity quantitatively, we calculated the output spectrum of the sensor (Fig. 4). A pair of single-mode photonic crystal waveguides serves as light input/output. The top and the holes of the photonic crystal are filled with air, water, and two kinds of petroleum-oil-based liquid samples, the refractive indices of which are set at 1.0, 1.33, 1.456, and 1.495, respectively. As expected, there is no resonant peak for air infiltration in this wavelength range, whereas water and the other two oil samples show very clear resonances in the spectra. A change in refractive index of $\Delta n=0.12$ between water and oil sample 1 results in a spectral shift of $21.3 \mathrm{~nm}$ (from 1454.00 to $1475.3 \mathrm{~nm}$ ) in our simulation. The subtle difference between the two oil samples of $\Delta n=0.039$

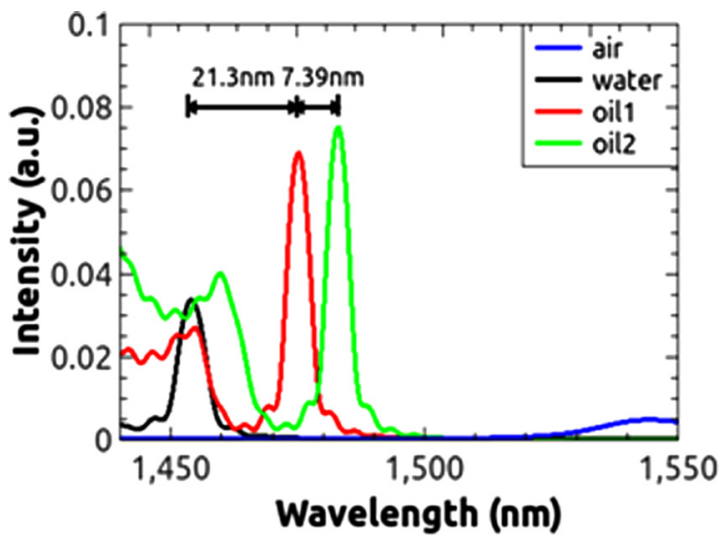

FIG. 4. Calculated transmission spectra of the sensor with analyte infiltrations. Three resonant wavelengths are 1454, 1475.3, and $1482.69 \mathrm{~nm}$ for water and oil sample infiltrations. A change in refractive index of $\Delta n=0.12$ between water and oil sample 1 results in a spectral shift of $21.3 \mathrm{~nm}$. The shift between the two oil samples is $7.39 \mathrm{~nm}$ for $\Delta n=0.039$ in refractive index.

induces a theoretical shift of $7.39 \mathrm{~nm}$ (from 1475.3 to $1482.69 \mathrm{~nm})$. The sensitivity of our current sensor has been significantly improved over that of a previous design with no slots in the cavity. For the previous design, a change in refractive index of $\Delta n=0.12$ between water and oil sample 1 results in spectral shifts of $10.4 \mathrm{~nm}$ in simulation and $12.4 \mathrm{~nm}$ in experiment as reported in Ref. 20.

The photonic crystal sensor was fabricated on a siliconon-insulator (SOI) wafer with a 260-nm-thick silicon slab and a $2-\mu \mathrm{m}$-thick buried silicon-dioxide layer. The patterns were first written in a 120-nm-thick Zep520 resist atop the silicon slab by a Leica EBPG 5000+ e-beam lithography system operating at $100 \mathrm{keV}$. Inductively coupling plasma (ICP) etching techniques was then used to etch the exposed areas. A gas flow rate of $\mathrm{SF}_{6} / \mathrm{O}_{2}$ and the etching time are controlled precisely to achieve a good depth-to-width ratio,

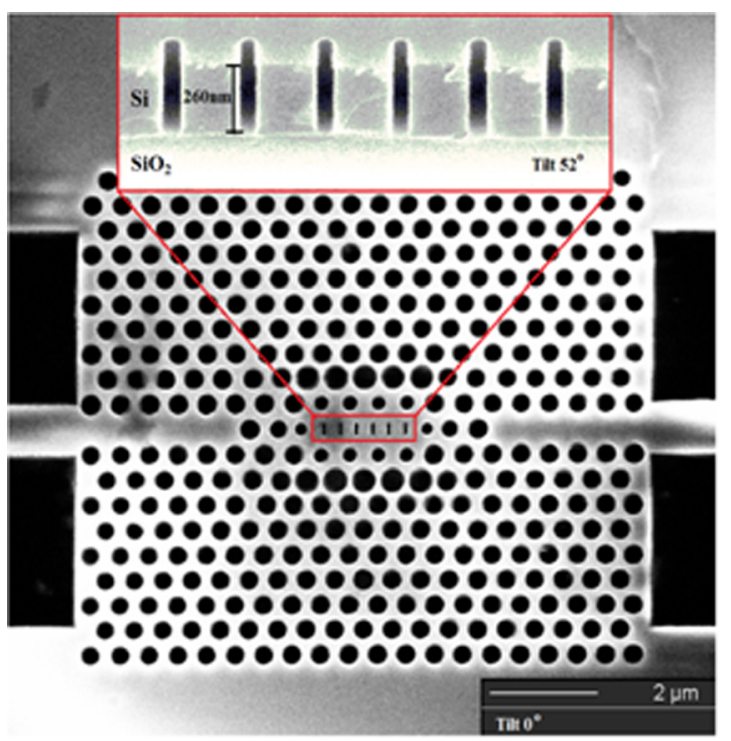

FIG. 5. Scanning electron microscope image of a sensor. Holes and slots are etched through a 260 -nm-thick silicon slab placed atop a buried $\mathrm{SiO}_{2}$ layer. A cross-section view of the slots of a test sample with a tilt angle of $52^{\circ}$ is shown in the inset. A good depth-to-width ratio is obtained by carefully controlling gas flow rates during ICP etching. 


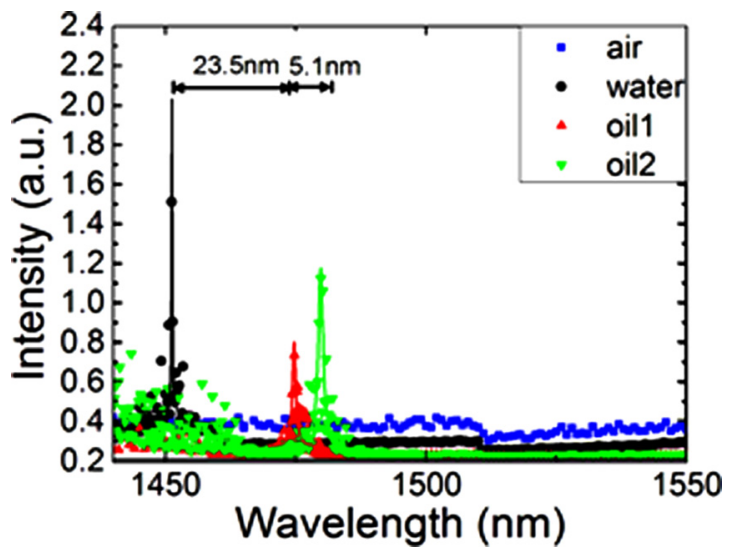

FIG. 6. Experimental transmission spectra of the sensor. The blue, black, red, and green spots were plotted for air, water, and oil sample infiltration. A change in refractive index of $\Delta n \sim 0.12$ between water and oil sample 1 results in a spectral shift of $23.5 \mathrm{~nm}$. The shift between two oil samples is $5.1 \mathrm{~nm}$ for $\Delta n \sim 0.039$ in refractive index.

as shown in the inset of Fig. 5. The remaining ZEP520 was ultimately dissolved in acetone.

The completed sensor is shown in Fig. 5. Light was coupled into and out of the sensor via a pair of traditional tapered strip waveguides. In order to minimize the mode mismatch, the strip waveguides are tapered from a width of $2.5 \mu \mathrm{m}$ at the edge to $866 \mathrm{~nm}$ at the interface of the photonic crystal waveguides. A tunable laser ranging from 1440 to $1630 \mathrm{~nm}$ serves as the light source. A TE polarizationmaintained lensed fiber is used to couple light from the laser to the input waveguide. Output light was collected by a group of objective lenses connected to a detector. Drops of liquid analytes were placed directly on the sensor with a syringe. After measurement, the liquid drops were blown off the chip with nitrogen gas.

Measured transmission spectra of our sensor are plotted in Fig. 6. Original data recorded by the optical detector (in solid spots) and their Lorentz fittings (in-lines) are both shown. The black spectrum, which corresponds to water sensing, shows a Q factor of 8060 at around $1451.2 \mathrm{~nm}$. The red spectrum was obtained after the oil sample 1 was placed on the sensor, and its Q factor is 1980 at $1474.7 \mathrm{~nm}$. Oil sample 2 has a spectrum shift of $5.1 \mathrm{~nm}$ from oil sample 1 with a $\mathrm{Q}$ factor of 1380. There is no obvious resonance for air sensing. In the presence of increased refractive indices, the resonant wavelength shifts are $23.5 \mathrm{~nm}$ for water/oil sample 1 and $5.1 \mathrm{~nm}$ for the two kinds of oil samples in the experiments. Comparing the simulation results of 21.3 and $7.39 \mathrm{~nm}$ for the same index changes, we observe excellent agreement between experimental data and theoretical simulations.
It should be noted that the oil samples we used in these experiments are real petroleum-based oil. Owing to complicated chemical compositions of the petroleum oil samples, their optical dispersion relations are difficult to predict precisely. The difference in index of $\Delta n=0.12$ for water/oil sample 1 and $\Delta n=0.039$ for the two oil samples are given at a single wavelength of $1550 \mathrm{~nm}$, though these numbers may vary slightly at other wavelengths. However, we do believe that a good index sensing has been achieved with the optimized photonic crystal sensor design.

In summary, we have designed a photonic-crystal-based sensor for higher refractive index sensing. We proved in both theory and experiment that our sensor can achieve high quality factors, high sensitivities, and high signal-to-noise ratios. Modifying the geometry of cavity and introducing inside slots has proved to be a rather effective method to enhance light-matter interaction. We believe our sensor is very promising for practical applications in bio/medical/ chemical sensing.

This work was supported by the project of FOM IPP IPOG-II: Nano Photonic Sensors (NPS).

${ }^{1}$ O. Levi, M. Lee, J. Zhang, V. Lousse, S. Brueck, S. Fan, and J. Harris, Proc SPIE 6447, 64470P (2007).

${ }^{2}$ M. Adams, G. DeRose, M. Loncar, and A. Scherer, J. Vac. Sci. Technol., B 23(6), 3168-3173 (2005).

${ }^{3}$ S. Johnson, S. Fan, P. Villeneuve, and J. Joannopoulos, Phys. Rev. B 60, 5751-5758 (1999).

${ }^{4}$ T. Krauss and R. De La Rue, Prog. Quantum Electron. 23, 51-96 (1999).

${ }^{5}$ N. Mortensen, S. Xiao, and J. Pedersen, Microfluid. Nanofluid. 4(1-2), $117-127$ (2008).

${ }^{6}$ C. Kang, C. Phare, Y. Vlasov, S. Assefa, and S. Weiss, Opt. Express 18(26), 27930-27937 (2010).

${ }^{7}$ X. Serey, S. Mandal, and D. Erickson, Nanotechnology 21, 305202 (2010).

${ }^{8}$ D. Dorfner, T. Zabel, T. Hurlimann, N. Hauke, L. Frandsen, U. Rant, G. Abstreiter, and J. Finley, Biosens. Bioelectron. 24, 3688-3692 (2009).

${ }^{9}$ E. Guillermain and P. Fauchet, Proc SPIE 7167, 71670D (2009).

${ }^{10}$ S. Buswell, V. Wright, J. Buriak, V. Van, and S. Evoy, Opt. Express 16(20), 15949-15957 (2008).

${ }^{11}$ Y. Akahane, T. Asano, B. Song, and S. Noda, Nature 425(6961), 944-947 (2003).

${ }^{12}$ Y. Akahane, T. Asano, B. Song, and S. Noda, Opt. Express 13(4), 1202-1214 (2005).

${ }^{13}$ B. Song, S. Noda, T. Asano, and Y. Akahane, Nat. Mater. 4(3), 207-210 (2005).

${ }^{14}$ T. Asano, B. Song, and S. Noda, Opt. Express 14(5), 1996-2002 (2006).

${ }^{15}$ M. Loncar, A. Scherer, and Y. Qiu, Appl. Phys. Lett. 82(26), 4648-4650 (2003).

${ }^{16}$ M. Adams, M. Loncar, A. Scherer, and Y. Qiu, IEEE J. Sel. Areas Commun. 23(7), 1348-1354 (2005).

${ }^{17}$ J. Berenger, J. Comput. Phys. 114(2), 185-200 (1994).

${ }^{18}$ V. Mandelshtam and H. Taylor, J. Chem. Phys. 107(17) 6756-6770 (1997).

${ }^{19}$ Y. Liu and H. Salemink, Opt. Express 20(18), 19912-19920 (2012).

${ }^{20}$ Y. Liu and H. Salemink, Europhys. Lett. 107, 34008 (2014). 